\title{
Impulsive Fractional Integrodifferential Equations and Lyapunov Method for Existence of Almost Periodic Solutions
}

\author{
Gani Tr. Stamov \\ Department of Mathematics and Physics, Technical University of Sofia, Burgasko Shose 59, 8800 Sliven, Bulgaria \\ Correspondence should be addressed to Gani Tr. Stamov; gstamov@abv.bg
}

Received 20 May 2015; Accepted 7 September 2015

Academic Editor: Sabri Arik

Copyright (C) 2015 Gani Tr. Stamov. This is an open access article distributed under the Creative Commons Attribution License, which permits unrestricted use, distribution, and reproduction in any medium, provided the original work is properly cited.

\begin{abstract}
The plan of this paper is to find conditions for the existence of almost periodic solutions for a class of impulsive fractional integrodifferential equations. The investigations are carried out by using a new fractional comparison principle, coupled with the fractional Lyapunov method. The stability behavior of the almost periodic solutions is also considered, extending the corresponding theory of impulsive integrodifferential equations.
\end{abstract}

\section{Introduction}

The states of many evolutionary processes are often subject to instantaneous perturbations and experience abrupt changes at certain moments of time. The duration of the changes is very short and negligible in comparison with the duration of the process considered and can be thought of as "momentarily" changes or as impulses. Systems with shortterm perturbations are often naturally described by impulsive differential equations. Such equations have become an active research subject in nonlinear science and have attracted more attention in many fields due to their importance in many branches of science and industry [1-3]. A great progress in studying impulsive integrodifferential equations has also been made. See, for example, [4-8] and the references therein. Indeed, impulsive integrodifferential systems played a very important role in modern applied mathematical models of real processes arising in phenomena studied in diverse disciplines.

Since the tools of impulsive fractional differential equations are applicable to various fields of study, the investigation of the theory of such equations has been started quite recently. In a series of papers different questions of the fundamental and qualitative theories of such equations have been investigated [9-14].

Additionally, in relation to the mathematical simulation in chaos, fluid dynamics, and many physical systems, recently the investigation of impulsive fractional functional differential equations began. Fractional-order impulsive functional differential equations are found to be more adequate than integer-order models in many applications and real-world phenomena studying in physics, mechanics, chemistry, engineering, and finance [15-18].

The problems of existence and uniqueness of the solutions of impulsive integrodifferential systems of fractional order have been studied by several authors. For example, Anguraj and Maheswari [19] proved the existence and uniqueness results for a fractional impulsive neutral integrodifferential system with infinite delay. By using Schaefer fixed point theorem, Gao et al. [20] established sufficient conditions for the existence and uniqueness of solutions for a class of impulsive integrodifferential equations with nonlocal conditions involving the Caputo fractional derivative. In the paper [21] Xie investigated the existence and uniqueness of mild solutions for impulsive fractional integrodifferential evolution equations with infinite delay in Banach spaces. However, to the best of our knowledge, there has not been any work so far considering the qualitative theory of such equations, and our aim here is mainly to fill the gap.

In the present paper, the problems of existence and stability of almost periodic solutions of impulsive integrodifferential equations of Caputo fractional order with impulse effect at fixed moments are considered by means of Lyapunov direct method. The paper is organized as follows. In Section 2 
we give some notations, the problem investigated in this paper is formulated, and some definitions are presented. In Section 3, we state and prove our main almost periodicity results, extending the corresponding theory of Caputo fractional order systems. We also establish stability criteria for the almost periodic solutions using the new fractional Lyapunov method. In Section 4 a fractional neural network system is considered as an example to demonstrate the application of our results. The concluding remarks are made in Section 5.

\section{Preliminaries}

Let $\mathbb{R}^{n}$ be the $n$-dimensional Euclidean space with norm $\|\cdot\|$, $\mathbb{R}=(-\infty, \infty), \mathbb{R}_{+}=[0, \infty)$, and $B_{\alpha}=\left\{x \in \mathbb{R}^{n}:\|x\| \leq \alpha\right\}$, $\alpha=$ const $>0$. By $\mathscr{B}, \mathscr{B}=\left\{\left\{t_{k}\right\}: t_{k} \in(-\infty, \infty), t_{k}<t_{k+1}\right.$, $\left.k= \pm 1, \pm 2, \ldots, \lim _{k \rightarrow \pm \infty} t_{k}= \pm \infty\right\}$ we denote the set of all sequences unbounded and strictly increasing with distance $\rho\left(\left\{t_{k}^{(1)}\right\},\left\{t_{k}^{(2)}\right\}\right)$.

Definition 1 (see [22]). For any $t \geq t_{0}, t_{0} \in \mathbb{R}$, Caputo's fractional derivative of order $q, 0<q<1$ with the lower limit $t_{0}$ for a function $l \in C^{1}\left[\left[t_{0}, b\right], \mathbb{R}^{n}\right], b>t_{0}$, is defined as

$$
{ }_{t_{0}}^{c} D_{t}^{q} l(t)=\frac{1}{\Gamma(1-q)} \int_{t_{0}}^{t} \frac{l^{\prime}(s)}{(t-s)^{q}} d s .
$$

Here and in what follows $\Gamma$ denotes the Gamma function.

Consider the following system of impulsive fractional integrodifferential equations:

$$
\begin{aligned}
{ }_{t_{0}}^{c} D_{t}^{q} x(t) & =f\left(t, x(t), \int_{t_{0}}^{t} K(t, \tau, x(\tau)) d \tau\right), \quad t \neq t_{k}, \\
\Delta x\left(t_{k}\right) & =I_{k}\left(x\left(t_{k}\right)\right), \quad k= \pm 1, \pm 2, \ldots,
\end{aligned}
$$

where $t_{0} \in \mathbb{R}, f \in C\left[\mathbb{R} \times \mathbb{R}^{n} \times \mathbb{R}^{n}, \mathbb{R}^{n}\right], K \in\left[\mathbb{R} \times \mathbb{R} \times \mathbb{R}^{n}, \mathbb{R}^{n}\right]$, $\left\{t_{k}\right\} \in \mathscr{B}, \Delta x\left(t_{k}\right)=x\left(t_{k}^{+}\right)-x\left(t_{k}\right)$, and $I_{k}: \mathbb{R}^{n} \rightarrow \mathbb{R}^{n}, k=$ $\pm 1, \pm 2, \ldots$

Let $x_{0} \in \mathbb{R}^{n}$. Denote by $x(t)=x\left(t ; t_{0}, x_{0}\right)$ the solution of system (2), satisfying the initial condition:

$$
x\left(t_{0}^{+}\right)=x_{0} .
$$

The solution $x(t)=x\left(t ; t_{0}, x_{0}\right)$ of problem (2), (3) is a piecewise continuous function [18] with points of discontinuity at the moments $t_{k}, k= \pm 1, \pm 2, \ldots$ at which it is continuous from the left; that is, the following relations are valid:

$$
\begin{aligned}
& x\left(t_{k}^{-}\right)=x\left(t_{k}\right), \\
& x\left(t_{k}^{+}\right)=x\left(t_{k}\right)+I_{k}\left(x\left(t_{k}\right)\right),
\end{aligned}
$$

$$
k= \pm 1, \pm 2, \ldots
$$

For some basic concepts and theorems of the solutions of impulsive fractional integrodifferential systems, we refer the reader to [19-21] and the references therein.

Let $P C=P C\left[\mathbb{R}, \mathbb{R}^{n}\right]=\left\{\psi: \mathbb{R} \rightarrow \mathbb{R}^{n}, \varphi\right.$ is piecewise continuous function with points of discontinuity of the first kind $t_{k},\left\{t_{k}\right\} \in \mathscr{B}$ at which $\psi\left(t_{k}^{-}\right)$and $\psi\left(t_{k}^{+}\right)$exist, and $\psi\left(t_{k}^{-}\right)=$ $\left.\psi\left(t_{k}\right)\right\}$.

Since the solutions of (2) belong to the space $P C$, what follows are definitions for almost periodicity [6]. Before stating these definitions, here are some essential notations.

For $\bar{T}, \bar{P} \in \mathscr{B}$, let seq $(\bar{T} \cup \bar{P}): \mathscr{B} \rightarrow \mathscr{B}$ be a map such that the set $\operatorname{seq}(\bar{T} \cup \bar{P})$ forms a strictly increasing sequence. For $D \subset \mathbb{R}$, let $\theta_{\varepsilon}(D)=\{t+\varepsilon, t \in D\}$ and $F_{\varepsilon}(D)=\cap\left\{\theta_{\varepsilon}(D)\right\}$ for $\varepsilon>0$. By $\Psi=(\psi(t), \bar{T})$, we shall denote the element from the space $P C \times \mathscr{B}$. For every sequence of real numbers $\left\{s_{n}\right\}$, $n=1,2, \ldots, \theta_{s_{n}} \Psi$ mean the sets $\left\{\psi\left(t+s_{n}\right), \bar{T}-s_{n}\right\} \subset P C \times \mathscr{B}$, where $\bar{T}-s_{n}=\left\{t_{k}-s_{n}, k= \pm 1, \pm 2, \ldots, n=1,2, \ldots\right\}$.

Definition 2 (see [6]). Let $\left\{t_{k}\right\} \in \mathscr{B}$.

(1) The set of sequences $\left\{t_{k}^{l}\right\}, t_{k}^{l}=t_{k+l}-t_{k}, k= \pm 1, \pm 2, \ldots$, $l=1,2, \ldots$, is said to be uniformly almost periodic, if from each infinite sequence of shifts $\left\{t_{k}-s_{n}\right\}, k= \pm 1, \pm 2, \ldots, n=$ $1,2, \ldots, s_{n} \in \mathbb{R}$ we can choose a subsequence convergent in $\mathscr{B}$.

(2) The sequence $\left\{\Psi_{n}\right\}, \Psi_{n}=\left(\psi_{n}(t), \bar{T}_{n}\right) \in P C\left[\mathbb{R}, \mathbb{R}^{n}\right] \times$ $\mathscr{B}$, is convergent to $\Psi$, where $\Psi=(\psi(t), \bar{T})$ and $(\psi(t), \bar{T}) \epsilon$ $P C\left[\mathbb{R}, \mathbb{R}^{n}\right] \times \mathscr{B}$, if and only if for any $\varepsilon>0$ there exists $n_{0}>0$ such that for $n \geq n_{0}$ it follows that

$$
\begin{array}{r}
\rho\left(\bar{T}, \bar{T}_{n}\right)<\varepsilon, \\
\left\|\psi_{n}(t)-\psi(t)\right\|<\varepsilon
\end{array}
$$

hold uniformly for $t \in \mathbb{R} \backslash F_{\varepsilon}\left(\operatorname{seq}\left(\bar{T}_{n} \cup \bar{T}\right)\right)$.

(3) The function $\psi \in P C\left[\mathbb{R}, \mathbb{R}^{n}\right]$ is said to be an almost periodic piecewise continuous function with points of discontinuity of the first kind $t_{k}$, if for every sequence of real numbers $\left\{s_{m}^{\prime}\right\}$ it follows that there exists a subsequence $\left\{s_{n}\right\}$, $s_{n}=s_{m_{n}}^{\prime}$, such that $\theta_{s_{n}} \Psi$ is compact in $P C\left[\mathbb{R}, \mathbb{R}^{n}\right] \times \mathscr{B}$.

By the specific character of system (2) we need the next definition.

Definition 3 (see [6]). The function $K \in C\left[\mathbb{R} \times \mathbb{R} \times \mathbb{R}^{n}, \mathbb{R}^{n}\right]$ is said to be integro-almost periodic in $t \in \mathbb{R}$ uniformly for $x \in \mathbb{R}^{n}$, if for every sequence of real numbers $\left\{s_{m}^{\prime}\right\}$ there exists a subsequence $\left\{s_{n}\right\}, s_{n}=s_{m_{n}}^{\prime}$, such that the sequence

$$
\left\{\int_{t_{0}}^{t+s_{n}} K\left(t+s_{n}, \tau, x(\tau)\right) d \tau\right\}
$$

converges uniformly with respect to $n \rightarrow \infty$.

Let $G_{k}=\left(t_{k-1}, t_{k}\right) \times \mathbb{R}^{n} \times \mathbb{R}^{n}, k= \pm 1, \pm 2, \ldots$, and $G=\cup_{k=-\infty}^{\infty} G_{k}$. In the further considerations, we shall use piecewise continuous auxiliary functions, which are analogous of the classical Lyapunov functions.

Definition 4. A function $V: \mathbb{R} \times \mathbb{R}^{n} \times \mathbb{R}^{n} \rightarrow \mathbb{R}_{+}$belongs to the class $V_{0}$, if one has the following:

(1) $V(t, x, y)$ is continuous in $G$ and locally Lipschitz continuous with respect to its second and third arguments on 
each of the sets $G_{k}$ with a Lipschitz constant $L>0$; that is, for $x_{1}, x_{2} \in \mathbb{R}^{n}, y_{1}, y_{2} \in \mathbb{R}^{n}$ and for $t \in \mathbb{R}$ it follows that

$$
\begin{aligned}
& \left|V\left(t, x_{1}, y_{1}\right)-V\left(t, x_{2}, y_{2}\right)\right| \\
& \quad \leq L\left(\left\|x_{1}-x_{2}\right\|+\left\|y_{1}-y_{2}\right\|\right) .
\end{aligned}
$$

(2) For each $k= \pm 1, \pm 2, \ldots$ and $x, y \in \mathbb{R}^{n}$, there exist the finite limits

$$
\begin{aligned}
& V\left(t_{k}^{-}, x, y\right)=\lim _{\substack{t \rightarrow t_{k} \\
t<t_{k}}} V(t, x, y), \\
& V\left(t_{k}^{+}, x, y\right)=\lim _{\substack{t \rightarrow t_{k} \\
t>t_{k}}} V(t, x, y),
\end{aligned}
$$

and $V\left(t_{k}^{-}, x, y\right)=V\left(t_{k}, x, y\right)$.

For a function $V \in V_{0}$ we define the following fractional order derivative (Dini-like derivative) in Caputo's sense.

Definition 5. Given a function $V \in V_{0}$, for $t \in\left[t_{k-1}, t_{k}\right), k=$ $\pm 1, \pm 2, \ldots$, and $x, y \in \mathbb{R}^{n}$, the upper right-hand derivative of $V$ in Caputo's sense of order $q, 0<q<1$, with respect to system (2) is defined by

$$
\begin{aligned}
& { }^{c} D_{+}^{q} V(t, x, y)=\lim _{\chi \rightarrow 0^{+}} \sup \frac{1}{\chi^{q}}[V(t, x, y)-V(t \\
& \quad-\chi, x-\chi^{q} f\left(t, x, \int_{t_{0}}^{t} K(t, \tau, x) d \tau\right), y \\
& \left.\left.\quad-\chi^{q} f\left(t, y, \int_{t_{0}}^{t} K(t, \tau, y) d \tau\right)\right)\right] .
\end{aligned}
$$

We shall also use the following class of functions:

$\mathscr{K}=\left\{a \in C\left[\mathbb{R}_{+}, \mathbb{R}_{+}\right]: a(r)\right.$ is strictly increasing and $a(0)=0\}$.

Introduce the following conditions:

(H2.1) The function $K(t, \tau, x)$ is integro-almost periodic in $t \in \mathbb{R}$ uniformly on $\tau \in \mathbb{R}$ and $x \in \mathbb{R}^{n}$.

(H2.2) The function $f\left(t, x, \int_{t_{0}}^{t} K(t, \tau, x) d \tau\right)$ is almost periodic in $t \in \mathbb{R}$ uniformly with respect to $x \in \mathbb{R}^{n}$.

(H2.3) The sequence $\left\{I_{k}(x)\right\}, k= \pm 1, \pm 2, \ldots$ is almost periodic uniformly with respect to $x \in \mathbb{R}^{n}$.

(H2.4) The set of sequences $\left\{t_{k}^{l}\right\}, t_{k}^{l}=t_{k+l}-t_{k}, k=$ $\pm 1, \pm 2, \ldots, l=1,2, \ldots$, is uniformly almost periodic, and $\inf _{k}\left\{t_{k}^{1}\right\}=\theta>0$.

Let conditions (H2.1)-(H2.4) hold, and let $\left\{s_{m}^{\prime}\right\}$ be an arbitrary sequence of real numbers. Then, there exists a subsequence $\left\{s_{n}\right\}, s_{n}=s_{m_{n}}^{\prime}$ such that system (2) moves to system

$$
\begin{aligned}
{ }_{t_{0}}^{c} D_{t}^{q} x(t)=f^{s}\left(t, x(t), \int_{t_{0}}^{t} K^{s}(t, \tau, x(\tau)) d \tau\right), & \\
& t \neq t_{k}^{s},
\end{aligned}
$$

We shall denote the set of systems of the type (10) by $H\left(f, K, I_{k}, t_{k}\right)$.

Now, we need a class of Lyapunov function connected with system (10).

Definition 6. The function $W: \mathbb{R} \times \mathbb{R}^{n} \rightarrow \mathbb{R}_{+}$belongs to class $W_{0}$; if $W(t, x)$ is continuous on $(t, x) \in \mathbb{R} \times \mathbb{R}^{n}, t \neq t_{k}^{s}$, $k= \pm 1, \pm 2, \ldots$, and $W(t, 0)=0, t \in \mathbb{R}$ and $W(t, x)$ is locally Lipschitz continuous with respect to its second argument, for $k= \pm 1, \pm 2, \ldots$, and $x \in \mathbb{R}^{n}$, there exist the finite limits

$$
\begin{aligned}
& W\left(t_{k}^{s-}, x\right)=\lim _{\substack{t \rightarrow t_{k}^{s} \\
t<t_{k}^{s}}} W(t, x), \\
& W\left(t_{k}^{s+}, x\right)=\lim _{\substack{t \rightarrow t_{k}^{s} \\
t>t_{k}^{s}}} W(t, x)
\end{aligned}
$$

and the equality $W\left(t_{k}^{s-}, x\right)=W\left(t_{k}^{s}, x\right)$ holds.

In the same way we can define a fractional order derivative (Dini-like derivative) in Caputo's sense for $W \in W_{0}$ as follows:

$$
\begin{aligned}
& { }^{c} D_{+}^{q} W(t, x)=\lim _{\chi \rightarrow 0^{+}} \sup \frac{1}{\chi^{q}}[W(t, x) \\
& \left.\quad-W\left(t-\chi, x-\chi^{q} f^{s}\left(t, x, \int_{t_{0}}^{t} K^{s}(t, \tau, x) d \tau\right)\right)\right] .
\end{aligned}
$$

Let $x_{1}^{*} \in \mathbb{R}^{n}$. Denote by $x_{1}(t)=x_{1}\left(t ; t_{0}, x_{1}^{*}\right)$ the solution of system (2), satisfying the initial condition $x_{1}\left(t_{0}^{+}\right)=x_{1}^{*}$. The next definitions are related to the stability of the solution of system (2).

Definition 7. The solution $x_{1}(t)$ of system (2) is said to be (a) stable, if

$$
\begin{gathered}
\left(\forall t_{0} \in \mathbb{R}\right) \\
(\forall \varepsilon>0) \\
(\exists \delta>0) \\
\left(\forall x_{0} \in \mathbb{R}^{n}:\left\|x_{0}-x_{1}^{*}\right\|<\delta\right) \\
\left(\forall t \geq t_{0}\right):\left\|x\left(t ; t_{0}, x_{0}\right)-x_{1}\left(t ; t_{0}, x_{1}^{*}\right)\right\|<\varepsilon ;
\end{gathered}
$$

(b) uniformly stable, if the number $\delta$ in (a) is independent of $t_{0} \in \mathbb{R}$

(c) attractive, if

$$
\begin{gathered}
\left(\forall t_{0} \in \mathbb{R}\right) \\
(\exists \lambda>0) \\
\left(\forall x_{0} \in \mathbb{R}^{n}:\left\|x_{0}-x_{1}^{*}\right\|<\lambda\right) \\
\lim _{t \rightarrow \infty} x\left(t ; t_{0}, x_{0}\right)=x_{1}\left(t ; t_{0}, x_{1}^{*}\right) ;
\end{gathered}
$$


(d) equiattractive, if

$$
\begin{gathered}
\left(\forall t_{0} \in \mathbb{R}\right) \\
(\exists \lambda>0) \\
(\forall \varepsilon>0) \\
(\exists T>0) \\
\left(\forall x_{0} \in \mathbb{R}^{n}:\left\|x_{0}-x_{1}^{*}\right\|<\lambda\right) \\
\left(\forall t \geq t_{0}+T\right):\left\|x\left(t ; t_{0}, x_{0}\right)-x_{1}\left(t ; t_{0}, x_{1}^{*}\right)\right\|<\varepsilon ;
\end{gathered}
$$

(e) uniformly attractive, if the numbers $\lambda$ and $T$ in (d) are independent of $t_{0} \in \mathbb{R}$;

(f) asymptotically stable, if it is stable and attractive;

(g) uniformly asymptotically stable, if it is uniformly stable and uniformly attractive;

(h) globally quasi-equiasymptotically stable, if

$$
\begin{gathered}
(\forall \lambda>0) \\
(\forall \varepsilon>0) \\
\left(\forall t_{0} \in \mathbb{R}\right) \\
(\exists T>0) \\
\left(\forall x_{0} \in \mathbb{R}^{n}:\left\|x_{0}-x_{1}^{*}\right\|<\lambda\right) \\
\left(\forall t \geq t_{0}+T\right):\left\|x\left(t ; t_{0}, x_{0}\right)-x_{1}\left(t ; t_{0}, x_{1}^{*}\right)\right\|<\varepsilon .
\end{gathered}
$$

Definition 8. The solutions of system (2) are

(a) equibounded, if

$$
\begin{gathered}
(\forall A>0) \\
\left(\forall t_{0} \in \mathbb{R}\right) \\
(\exists B>0) \\
\left(\forall x_{0} \in \mathbb{R}^{n}:\left\|x_{0}\right\|<A\right) \\
\left(\forall t \geq t_{0}\right):\left\|x\left(t ; t_{0}, x_{0}\right)\right\|<B ;
\end{gathered}
$$

(b) uniformly bounded, if the number B in (a) is independent of $t_{0} \in \mathbb{R}$.

Definition 9. The solution $x_{1}(t)$ of (2) is said to be globally perfectly uniform-asymptotically stable, if it is uniformly stable, the number $T$ in Definition $7(\mathrm{~h})$ is independent of $t_{0} \in \mathbb{R}$, and the solutions of (2) are uniformly bounded.

In the proof of the main results we shall use the following comparison lemma. As the proof is similar to that of Theorem 3.1 in [18], we omit it.

Lemma 10. Assume the following:

(1) The function $g:\left[t_{0}, \infty\right) \times \mathbb{R}_{+} \rightarrow \mathbb{R}$ is continuous in each of the sets $\left(t_{k-1}, t_{k}\right] \times \mathbb{R}_{+}, k= \pm 1, \pm 2, \ldots$.

(2) $B_{k} \in C\left[\mathbb{R}_{+}, \mathbb{R}_{+}\right]$and $\psi_{k}(u)=u+B_{k}(u) \geq 0, k=$ $\pm 1, \pm 2, \ldots$, are nondecreasing with respect to $u$.
(3) The maximal solution $u^{+}\left(t ; t_{0}, u_{0}\right)$ of the scalar problem

$$
\begin{aligned}
{ }_{t_{0}}^{c} D_{t}^{q} u(t) & =g(t, u(t)), \quad t \neq t_{k}, \\
u\left(t_{0}\right) & =u_{0} \geq 0, \\
\Delta u\left(t_{k}\right) & =B_{k}\left(u\left(t_{k}\right)\right), \quad k= \pm 1, \pm 2, \ldots,
\end{aligned}
$$

is defined in the interval $\left[t_{0}, \infty\right)$.

(4) The function $V \in V_{0}$ is such that, for $t \in\left[t_{0}, \infty\right)$,

$$
\begin{aligned}
V\left(t^{+}, x+I_{k}(x), y+I_{k}(y)\right) \leq & \psi_{k}(V(t, x, y)), \\
& t=t_{k}, k= \pm 1, \pm 2, \ldots, \\
{ }^{c} D_{+}^{q} V(t, x, y) \leq & g(t, V(t, x, y)), \\
t & \neq t_{k}, \quad k= \pm 1, \pm 2, \ldots .
\end{aligned}
$$

Then $V\left(t_{0}, x_{0}, y_{0}\right) \leq u_{0}$ implies

$$
\begin{aligned}
V\left(t, x\left(t ; t_{0}, x_{0}\right), y\left(t ; t_{0}, y_{0}\right)\right) \leq u^{+}\left(t ; t_{0}, u_{0}\right) & \\
& t \in\left[t_{0}, \infty\right),
\end{aligned}
$$

where $x=x\left(t ; t_{0}, x_{0}\right), y=y\left(t ; t_{0}, y_{0}\right)$ are two solutions of (2), $x_{0}, y_{0} \in \mathbb{R}^{n}$, existing on $\left[t_{0}, \infty\right)$.

\section{Main Results}

Theorem 11. Assume the following:

(1) Conditions (H2.1)-(H2.4) are met.

(2) There exist functions $V \in V_{0}$ and $a, b \in \mathscr{K}$ such that

$$
\begin{aligned}
& a(\|x-y\|) \leq V(t, x, y) \leq b(\|x-y\|), \\
& (t, x, y) \in \mathbb{R} \times \mathbb{R}^{n} \times \mathbb{R}^{n}, \\
& V\left(t_{k}^{+}, x+I_{k}(x), y+I_{k}(y)\right) \leq V\left(t_{k}, x, y\right), \\
& (x, y) \in \mathbb{R}^{n} \times \mathbb{R}^{n}, k= \pm 1, \pm 2, \ldots, \\
& { }^{c} D_{+}^{q} V(t, x, y) \leq-M V(t, x, y), \\
& (t, x, y) \in G, M>0 .
\end{aligned}
$$

(3) There exists a solution $x(t)=x\left(t ; t_{0}, x_{0}\right)$ of (2) such that $x(t) \in B_{\alpha}$, where $t \geq t_{0}, \alpha>0$.

Then for system (2) there exists a unique almost periodic solution $\omega(t)$ such that

(1) $\omega(t) \in B_{\alpha_{1}}, \alpha_{1}<\alpha$.

(2) $\omega(t)$ is globally perfectly uniform-asymptotically stable.

(3) $H\left(\omega, t_{k}\right) \subset H\left(f, K, I_{k}, t_{k}\right)$

Proof. Let $t_{0} \in \mathbb{R}$ and let $\left\{s_{p}\right\}$ be any sequence of real numbers such that $s_{p} \rightarrow \infty$ as $p \rightarrow \infty$ and $\left\{s_{p}\right\}$ moves system (2), (3) to a system at $H\left(f, K, I_{k}, t_{k}\right)$.

For any real number $\beta$, let $p_{0}=p_{0}(\beta)$ be the smallest value of $p$, such that $s_{p_{0}}+\beta \geq t_{0}$. Since $x(t) \in B_{\alpha_{1}}, \alpha_{1}<\alpha$, for all $t \geq t_{0}$, then $x\left(t+s_{p}\right) \in B_{\alpha_{1}}$ for $t \geq \beta, p \geq p_{0}$. 
Let $U, U \subset(\beta, \infty)$ be a compact. Then, for any $\varepsilon>0$, choose an integer $n_{0}(\varepsilon, \beta) \geq p_{0}(\beta)$ so large that, for $l \geq p \geq$ $n_{0}(\varepsilon, \beta)$ and $t>t_{0}, t \neq t_{k}$, it follows that

$$
\begin{aligned}
& b\left(2 \alpha_{1}\right) E_{q}\left(-M\left(\beta+s_{p}-t_{0}\right)^{q}\right) \leq \frac{a(\varepsilon)}{2}, \\
& \| f\left(t+s_{l}, x, \int_{t_{0}}^{t+s_{l}} K\left(t+s_{l}, \tau, x\right) d \tau\right) \\
& \quad-f\left(t+s_{p}, x, \int_{t_{0}}^{t+s_{p}} K\left(t+s_{p}, \tau, x\right) d \tau\right) \| \\
& \quad \leq \frac{a(\varepsilon) M}{2 L},
\end{aligned}
$$

where $E_{q}(\cdot)$ is the corresponding Mittag-Leffler function [22].

Now, following [23], we shall set $m(\sigma)=V(\sigma, x(\sigma), x(\sigma+$ $\left.\left.s_{p}-s_{l}\right)\right)$ and $S(x, \chi, r, q)=\sum_{r=1}^{n}(-1)^{r+1}\left(\begin{array}{c}q \\ r\end{array}\right) x(t-r \chi)$. Then, for small $\chi>0$, we have

$$
\begin{aligned}
& V\left(\sigma, x(\sigma), x\left(\sigma+s_{p}-s_{l}\right)\right)-V\left(\sigma-\chi, S_{1}, S_{2}\right) \\
& \quad=V\left(\sigma, x(\sigma), x\left(\sigma+s_{p}-s_{l}\right)\right)-V(\sigma-\chi, x(\sigma)
\end{aligned}
$$

$$
\begin{aligned}
& -\chi^{q} f\left(\sigma, x(\sigma), \int_{t_{0}}^{\sigma} K(\sigma, \tau, x(\tau)) d \tau\right), x\left(\sigma+s_{p}\right. \\
& \left.-s_{l}\right)-\chi^{q} f\left(\sigma+s_{p}-s_{l}, x\left(\sigma+s_{p}-s_{l}\right),\right. \\
& \left.\left.\int_{t_{0}}^{\sigma+s_{p}-s_{l}} K\left(\sigma+s_{p}-s_{l}, \tau, x(\tau)\right) d \tau\right)\right)+V(\sigma-\chi, \\
& x(\sigma)-\chi^{q} f\left(\sigma, x(\sigma), \int_{t_{0}}^{\sigma} K(\sigma, \tau, x(\tau)) d \tau\right), x(\sigma \\
& \left.+s_{p}-s_{l}\right)-\chi^{q} f\left(\sigma+s_{p}-s_{l}, x\left(\tau+s_{p}-s_{l}\right),\right. \\
& \left.\left.\int_{t_{0}}^{\sigma+s_{p}-s_{l}} K\left(\sigma+s_{p}-s_{l}, \tau, x(\tau)\right) d \tau\right)\right)-V(\sigma-\chi, \\
& \left.S_{1}, S_{2}\right),
\end{aligned}
$$

where $S_{1}=S(x(\sigma), \chi, r, q)$ and $S_{2}=S\left(x\left(\sigma+s_{p}-s_{l}\right), \chi, r, q\right)$.

Then by using the fractional order derivative of $V$ in Caputo's sense and (24) we obtain

$$
\begin{aligned}
& { }^{c} D_{+}^{q} m(\sigma)=\lim _{\substack{x \rightarrow 0 \\
n \chi=-t t_{0}}} \sup \frac{1}{\chi^{q}}\left[V\left(\sigma, x(\sigma), x\left(\sigma+s_{p}-s_{l}\right)\right)\right. \\
& -V\left(\sigma-\chi, x(\sigma)-\chi^{q} f\left(\sigma, x(\sigma), \int_{t_{0}}^{\sigma} K(\sigma, \tau, x(\tau)) d \tau\right), x\left(\sigma+s_{p}-s_{l}\right)-\chi^{q} f\left(\sigma+s_{p}-s_{l}, x\left(\sigma+s_{p}-s_{l}\right), \int_{t_{0}}^{\sigma+s_{p}-s_{l}} K\left(\sigma+s_{p}-s_{l}, \tau, x(\tau)\right) d \tau\right)\right) \\
& +V\left(\sigma-\chi, x(\sigma)-\chi^{q} f\left(\sigma, x(\sigma), \int_{t_{0}}^{\sigma} K(\sigma, \tau, x(\tau)) d \tau\right), x\left(\sigma+s_{p}-s_{l}\right)-\chi^{q} f\left(\sigma+s_{p}-s_{l}, x\left(\sigma+s_{p}-s_{l}\right), \int_{t_{0}}^{\sigma+s_{p}-s_{l}} K\left(\sigma+s_{p}-s_{l}, \tau, x(\tau)\right) d \tau\right)\right) \\
& \left.\quad-V\left(\sigma-\chi, s_{1}, s_{2}\right)\right] \leq-M V\left(\sigma, x(\sigma), x\left(\sigma+s_{p}-s_{l}\right)\right)+L \| f\left(\sigma+s_{p}-s_{l}, x\left(\sigma+s_{p}-s_{l}\right), \int_{t_{0}}^{\sigma+s_{p}-s_{l}} K\left(\sigma+s_{p}-s_{l}, \tau, x(\tau) d \tau\right)\right)-f\left(\sigma, x\left(\sigma+s_{p}\right.\right. \\
& \left.\left.-s_{l}\right), \int_{t_{0}}^{\sigma} K(\sigma, \tau, x(\tau) d \tau)\right) \| .
\end{aligned}
$$

Set $\zeta=\sigma-s_{p}$. Then,

$$
\begin{aligned}
& f\left(\sigma+s_{p}-s_{l}, x\left(\sigma+s_{p}-s_{l}\right),\right. \\
& \left.\quad \int_{t_{0}}^{\sigma+s_{p}-s_{l}} K\left(t+s_{p}-s_{l}, \tau, x(\tau) d \tau\right)\right)-f(\sigma \\
& \left.x\left(\sigma+s_{p}-s_{l}\right), \int_{t_{0}}^{\sigma} K(t, \tau, x(\tau) d \tau)\right)=f\left(\zeta+s_{l},\right. \\
& \left.\quad x\left(\zeta+s_{l}\right), \int_{t_{0}}^{\zeta+s_{l}} K\left(\zeta+s_{l}, \tau, x(\tau) d \tau\right)\right)-f\left(\zeta+s_{p},\right. \\
& \left.\quad x\left(\zeta+s_{p}\right), \int_{t_{0}}^{\zeta+s_{p}} K\left(\zeta+s_{p}, \tau, x(\tau) d \tau\right)\right)
\end{aligned}
$$

and by (24)

$$
\begin{aligned}
& { }^{c} D_{+}^{q} V\left(\sigma, x(\sigma), x\left(\sigma+s_{p}-s_{l}\right)\right) \\
& \quad \leq-M V\left(\sigma, x(\sigma), x\left(\sigma+s_{p}-s_{l}\right)\right)+\frac{a(\varepsilon) M}{2} .
\end{aligned}
$$

On the other hand, from $\sigma=t_{k}-\left(s_{l}-s_{p}\right)$, it follows that

$$
\begin{aligned}
& V\left(\sigma, x(\sigma)+I_{k}(x(\sigma)), x\left(\sigma+s_{l}-s_{p}\right)\right. \\
& \left.\quad+I_{k}\left(x\left(\sigma+s_{l}-s_{p}\right)\right)\right) \\
& \quad \leq V\left(\sigma, x(\sigma), x\left(\sigma+s_{l}-s_{p}\right)\right) .
\end{aligned}
$$

Then from (28) and (29) it follows that the conditions of Lemma 10 are fulfilled and consequently, for $p, l \geq n_{0}(\varepsilon, \beta)$ and any $t \in U$,

$$
\begin{aligned}
& V\left(t+s_{p}, x\left(t+s_{p}\right), x\left(t+s_{l}\right)\right) \\
& \quad \leq E_{q}\left(-M\left(t+s_{p}-s_{l}\right)^{q}\right) \\
& \quad \cdot V\left(t_{0}, x\left(t_{0}+s_{p}\right), x\left(t_{0}+s_{l}\right)\right)+\frac{a(\varepsilon)}{2} \leq a(\varepsilon) .
\end{aligned}
$$

Finally, from (21) for any $t \in U$, we get

$$
\left\|x\left(t+s_{p}\right)-x\left(t+s_{l}\right)\right\|<\varepsilon .
$$


Consequently, there exists a function $\omega(t)$, such that $x(t+$ $\left.s_{p}\right)-\omega(t) \rightarrow \infty$ for $p \rightarrow \infty$. Since $\beta$ is arbitrary, it follows that $\omega(t)$ is defined uniformly on $t \in \mathbb{R}$. Next, we shall show that $\omega(t)$ is a solution of (10).

Since $x\left(t ; t_{0}, x_{0}\right)$ is a solution of (2), (3), we have

$$
\begin{aligned}
& \|\|_{t_{0}}^{c} D_{t}^{q} x\left(t+s_{p}\right)-{ }_{t_{0}}^{c} D_{t}^{q} x\left(t+s_{l}\right)\|\leq\| f(t \\
& \left.+s_{p}, x\left(t+s_{p}\right), \int_{t_{0}}^{t+s_{p}} K\left(t+s_{p}, \tau, x\right) d \tau\right)-f(t \\
& \left.+s_{l}, x\left(t+s_{p}\right), \int_{t_{0}}^{t+s_{l}} K\left(t+s_{l}, \tau, x(\tau)\right) d \tau\right) \| \\
& +\| f(t \\
& \left.\quad+s_{l}, x\left(t+s_{p}\right), \int_{t_{0}}^{t+s_{l}} K\left(t+s_{l}, \tau, x(\tau)\right) d \tau\right)-f(t \\
& \left.+s_{l}, x\left(t+s_{l}\right), \int_{t_{0}}^{t+s_{l}} K\left(t+s_{l}, \tau, x(\tau)\right) d \tau\right) \|,
\end{aligned}
$$

for $t+s_{\kappa} \neq t_{k}, \kappa=p, l ; k= \pm 1, \pm 2, \ldots$.

As $x\left(t+s_{p}\right) \in B_{\alpha_{1}}$ for large $s_{p}$ and for each compact subset of $\mathbb{R}$ there exists $n_{1}(\varepsilon)>0$ such that if $l \geq p \geq n_{1}(\varepsilon)$, then

$$
\begin{aligned}
& \| f\left(t+s_{p}, x\left(t+s_{p}\right), \int_{t_{0}}^{t+s_{p}} K\left(t+s_{p}, \tau, x(\tau)\right) d \tau\right) \\
& \quad-f(t \\
& \left.\quad+s_{l}, x\left(t+s_{p}\right), \int_{t_{0}}^{t+s_{l}} K\left(t+s_{l}, \tau, x(\tau)\right) d \tau\right) \|<\frac{\varepsilon}{2} .
\end{aligned}
$$

Since $x\left(t+s_{\kappa}\right) \in B_{\beta(\alpha)}, \kappa=p, l$, then it follows that there exists $n_{2}(\varepsilon)>0$ such that if $l \geq p \geq n_{2}(\varepsilon)$, then

$$
\begin{aligned}
& \| f\left(t+s_{l}, x\left(t+s_{p}\right), \int_{t_{0}}^{t+s_{l}} K\left(t+s_{l}, \tau, x(\tau)\right) d \tau\right) \\
& \quad-f(t \\
& \left.\quad+s_{l}, x\left(t+s_{l}\right), \int_{t_{0}}^{t+s_{l}} K\left(t+s_{l}, \tau, x(\tau)\right) d \tau\right) \|<\frac{\varepsilon}{2} .
\end{aligned}
$$

For $l \geq p \geq n(\varepsilon), n(\varepsilon)=\max \left\{n_{1}(\varepsilon), n_{2}(\varepsilon)\right\}$, we obtain

$$
\left\|{ }_{t_{0}}^{c} D_{t}^{q} x\left(t+s_{p}\right)-{ }_{t_{0}}^{c} D_{t}^{q} x\left(t+s_{l}\right)\right\| \leq \varepsilon,
$$

where $t+s_{\kappa} \neq t_{k}^{s}$, which shows that $\lim _{p \rightarrow \infty t_{0}} D_{t}^{q} x\left(t+s_{p}\right)$ exists uniformly on all compact subsets of $\mathbb{R}$.
Let now $\lim _{p \rightarrow \infty}{ }_{t_{0}}^{c} D_{t}^{q} x\left(t+s_{p}\right)={ }_{t_{0}}^{c} D_{t}^{q} \omega(t)$, and

$$
\begin{aligned}
& { }_{t_{0}}^{c} D_{t}^{q} \omega(t)=\lim _{p \rightarrow \infty}[f(t \\
& \left.+s_{p}, x\left(t+s_{p}\right), \int_{t_{0}}^{t+s_{p}} K\left(t+s_{p}, \tau, x(\tau)\right) d \tau\right) \\
& \quad-f\left(t+s_{p}, \omega(t), \int_{t_{0}}^{t+s_{l}} K\left(t+s_{p}, \tau, \omega(\tau)\right) d \tau\right) \\
& \left.+f\left(t+s_{p}, \omega(t), \int_{t_{0}}^{t+s_{p}} K\left(t+s_{p}, \tau, \omega(\tau)\right) d \tau\right)\right] \\
& \quad=f^{s}\left(t, \omega(t), \int_{t_{0}}^{t} K^{s}(t, \tau, \omega(\tau)) d \tau\right),
\end{aligned}
$$

where $t \neq t_{k}^{s}, t_{k}^{s}=\lim _{p \rightarrow \infty} t_{k+p(s)}$.

On the other hand, for $t+s_{p}=t_{k}^{s}$, it follows that

$$
\begin{aligned}
& \omega\left(t_{k}^{s+}\right)-\omega\left(t_{k}^{s-}\right) \\
& \quad=\lim _{p \rightarrow \infty}\left(x\left(t_{k}^{s}+s_{p}+0\right)-x\left(t_{k}^{s}+s_{p}-0\right)\right) \\
& \quad=\lim _{p \rightarrow \infty} I_{k}^{s}\left(x\left(t_{k}^{s}+s_{p}\right)\right)=I_{k}^{s}\left(\omega\left(t_{k}^{s}\right)\right) .
\end{aligned}
$$

From (36) and (37), we get that $\omega(t)$ is a solution of (10). We shall show that $\omega(t)$ is an almost periodic function.

Let the sequence $\left\{s_{p}\right\}$ move system (2) to $H\left(f, K, I_{k}, t_{k}\right)$. For any $\varepsilon>0$, there exists $m_{0}(\varepsilon)>0$ such that if $l \geq p \geq$ $m_{0}(\varepsilon)$, then

$$
\begin{aligned}
& E_{q}\left(-M\left(s_{p}\right)^{q}\right) b\left(2 \alpha_{1}\right)<\frac{a(\varepsilon)}{4}, \\
& \| f\left(\sigma+s_{p}, x\left(\sigma+s_{p}\right), \int_{t_{0}}^{\sigma+s_{p}} K\left(\sigma+s_{p}, \tau, x(\tau)\right) d \tau\right) \\
& \quad-f(\sigma \\
& \left.\quad+s_{l}, x\left(\sigma+s_{l}\right), \int_{t_{0}}^{\sigma+s_{l}} K\left(\sigma+s_{l}, \tau, x(\tau)\right) d \tau\right) \| \\
& \quad<\frac{a(\varepsilon)}{4 L} .
\end{aligned}
$$

For each fixed $t \in \mathbb{R}$ let $\tau_{\varepsilon}=a(\varepsilon) / 4 L$ be a translation number of $f$ such that $t+\tau_{\varepsilon} \geq 0$. Consider the function

$$
V\left(\tau_{\varepsilon}+\sigma, \omega(\sigma), \omega\left(\sigma+s_{l}-s_{p}\right)\right)
$$

where $t \leq \sigma \leq t+s_{p}$. 
Then, in the same way like above, we have

$$
\begin{aligned}
& { }^{c} D_{+}^{q} V\left(\tau_{\varepsilon}+\sigma, \omega(\sigma), \omega\left(\sigma+s_{l}-s_{p}\right)\right) \leq-M V\left(\tau_{\varepsilon}\right. \\
& \left.+\sigma, \omega(\sigma), \omega\left(\sigma+s_{l}-s_{p}\right)\right)+L \| f^{s}(\sigma, \omega(\sigma), \\
& \left.\int_{t_{0}}^{\sigma} K^{s}(\sigma, \tau, \omega(\tau)) d \tau\right)-f^{s}\left(\tau_{\varepsilon}+\sigma, \omega(\sigma),\right. \\
& \left.\int_{t_{0}}^{\tau_{\varepsilon}+\sigma} K^{s}\left(\tau_{\varepsilon}+\sigma, \tau, \omega(\tau)\right) d \tau\right)\|+L\| f^{s}\left(\sigma+s_{l}\right. \\
& -s_{i}, \omega\left(\sigma+s_{l}-s_{p}\right), \\
& \left.\int_{t_{0}}^{\sigma+s_{l}-s_{p}} K^{s}\left(\sigma+s_{l}-s_{p}, \tau, \omega(\tau)\right) d \tau\right)-f^{s}\left(\tau_{\varepsilon}+\sigma\right. \\
& \left.\omega\left(\sigma+s_{l}-s_{p}\right), \int_{t_{0}}^{\tau_{\varepsilon}+\sigma} K^{s}\left(\tau_{\varepsilon}+\sigma, \tau, \omega(\tau)\right) d \tau\right) \| \\
& \leq-M V\left(\tau_{\varepsilon}+\sigma, \omega(\sigma), \omega\left(\sigma+s_{l}-s_{p}\right)\right)+\frac{3 a(\varepsilon)}{4} .
\end{aligned}
$$

On the other hand,

$$
\begin{aligned}
& V\left(\tau_{\varepsilon}+t_{k}^{s}, \omega\left(t_{k}^{s}\right)+I_{k}^{s}\left(\omega\left(t_{k}^{s}\right)\right), \omega\left(t_{k}^{s}+s_{l}-s_{p}\right)\right. \\
& \left.\quad+I_{k}^{s}\left(\omega\left(t_{k}^{s}+s_{l}-s_{p}\right)\right)\right) \leq V\left(\tau_{\varepsilon}\right. \\
& \left.\quad+t_{k}^{s}, \omega\left(t_{k}^{s}\right), \omega\left(t_{k}^{s}+s_{l}-s_{p}\right)\right) .
\end{aligned}
$$

From (40), (41), and Lemma 10 it follows that

$$
\begin{aligned}
& V\left(\tau_{\varepsilon}+t+s_{p}, \omega\left(t+s_{p}\right), \omega\left(t+s_{l}\right)\right) \\
& \leq E_{q}\left(-M\left(s_{p}\right)^{q}\right) V\left(\tau_{\varepsilon}+t, \omega(t), \omega\left(t+s_{p}-s_{l}\right)\right) \\
& \quad+\frac{3 a(\varepsilon)}{4}<a(\varepsilon) .
\end{aligned}
$$

Then, from (42), for $l \geq p \geq m_{0}(\varepsilon)$, we have

$$
\left\|\omega\left(t+s_{p}\right)-\omega\left(t+s_{l}\right)\right\|<\varepsilon .
$$

Now, from definitions of the sequence $\left\{s_{p}\right\}$ and for $l \geq p \geq$ $m_{0}(\varepsilon)$ it follows that $\rho\left(t_{k}+s_{p}, t_{k}+s_{l}\right)<\varepsilon$.

Then from (43) and the last inequality we obtain that the sequence $\omega\left(t+s_{p}\right)$ is convergent uniformly to the function $\omega(t)$. Assertions (1) and (3) of the theorem follow immediately. We shall prove assertion (2).

Let $\bar{\omega}(t)$ be an arbitrary solution of (10).
Set

$$
\begin{aligned}
& u(t)=\bar{\omega}(t)-\omega(t) \\
& g^{s}\left(t, u(t), \int_{t_{0}}^{t} K^{s}(t, \tau, u(\tau)) d \tau\right) \\
& =f^{s}\left(t, u(t)+\omega(t), \int_{t_{0}}^{t} K^{s}(t, \tau, u(\tau)+\omega(\tau)) d \tau\right) \\
& \quad-f^{s}\left(t, \omega(t), \int_{t_{0}}^{t} K^{s}(t, \tau, \omega(\tau)) d \tau\right) \\
& B_{k}^{s}(u)=I_{k}^{s}(u+\omega)-I_{k}^{s}(\omega)
\end{aligned}
$$

Now we consider system

$$
\begin{aligned}
{ }_{{ }_{0}}^{c} D_{t}^{q} u & =g^{s}(t, u(t)), \quad t \neq t_{k}^{s}, \\
\Delta u\left(t_{k}^{s}\right) & =B_{k}^{s}\left(u\left(t_{k}^{s}\right)\right), \quad k= \pm 1, \pm 2, \ldots,
\end{aligned}
$$

and let $W(t, u(t))=V(t, \omega(t), \omega(t)+u(t))$. Then, from Lemma 10 it follows that zero solution $u(t)=0$ of (45) is globally perfectly uniform-asymptotically stable, and consequently $\omega(t)$ is globally perfectly uniform-asymptotically stable.

\section{An Example}

Consider the following impulsive integrodifferential fractional-order cellular neural network $(\mathrm{CNN})$ :

$$
\begin{aligned}
{ }_{t_{0}}^{c} D_{t}^{q} x_{i}(t)= & -c_{i} x_{i}(t)+\sum_{j=1}^{n} a_{i j}(t) f_{j}\left(x_{j}(t)\right) \\
& +\sum_{j=1}^{n} \int_{t_{0}}^{t} k_{i j}(t, \tau) x_{j}(\tau) d \tau+I_{i}, \\
\Delta x_{i}\left(t_{k}\right)= & P_{i k} x_{i}\left(t_{k}\right), \quad k= \pm 1, \pm 2, \ldots,
\end{aligned}
$$

where $i=1,2, \ldots, n$. The study of impulsive CNNs and its applications can be found, for example, in $[6,7]$.

We suppose that for system (46) there exists a bounded solution and the following conditions are met:

(H4.1) The functions $a_{i j}(t)$ are almost periodic on $t$ in sense of Bohr, $k_{i j}(t, \tau), i, j=1,2, \ldots, n$, are integro-almost periodic in $t \in \mathbb{R}$, and there exist constants $L_{i}>0$ and $H_{i}>0$ such that

$$
\begin{gathered}
\left|f_{i}(u)-f_{i}(v)\right| \leq L_{i}|u-v|, \\
\left|f_{i}(u)\right| \leq H_{i}
\end{gathered}
$$$$
u, v \in \mathbb{R}, i=1,2, \ldots, n,
$$

$$
\min _{1 \leq i \leq n}\left(c_{i}-L_{i} \sum_{j=1}^{n}\left|a_{i j}\right|\right)=A>0
$$

for $t>t_{0}$. 
(H4.2) The sequences of constants $\left\{P_{i k}\right\}$ are almost periodic uniformly with $i=1,2, \ldots, n$ and

$$
-1<P_{i k} \leq 0
$$

for $i=1,2, \ldots, n, k= \pm 1, \pm 2, \ldots$.

Suppose that $t_{0} \in \mathbb{R}_{+}$and let $\left\{s_{p}\right\}$ be a sequence of real numbers defined as in the proof of Theorem 11. We define a Lyapunov function in the form

$$
V(x, y)=\sum_{i=1}^{n}\left|x_{i}-y_{i}\right| .
$$

Now, when $t=t_{k}$ by condition (H4.2) it follows that

$$
\begin{aligned}
& V\left(x\left(t_{k}^{+}+s_{p}\right), x\left(t_{k}^{+}+s_{l}\right)\right)=\sum_{i=1}^{n} \mid x_{i}\left(t_{k}^{+}+s_{p}\right) \\
& -x_{i}\left(t_{k}^{+}+s_{l}\right)\left|=\sum_{i=1}^{n}\right| x_{i}\left(t_{k}+s_{p}\right)+P_{i k} x_{i}\left(t_{k}+s_{p}\right) \\
& -x_{i}\left(t_{k}+s_{l}\right)-P_{i k} x_{i}\left(t_{k}+s_{l}\right) \mid \leq \sum_{i=1}^{n}\left(1+P_{i k}\right) \\
& \cdot\left|x_{i}\left(t_{k}+s_{p}\right)-x_{i}\left(t_{k}+s_{l}\right)\right| \leq V\left(x\left(t_{k}+s_{p}\right), x\left(t_{k}\right.\right. \\
& \left.\left.+s_{l}\right)\right) .
\end{aligned}
$$

On the other hand by the definition of $\left\{s_{p}\right\}$ it follows that $\sum_{i=1}^{n}\left|x_{i}\left(t+s_{p}\right)-x_{i}\left(t_{k}+s_{l}\right)\right|=v>0$. Then, for $M \in(0, A)$ and for any $\varepsilon, 0<\varepsilon<(A-M) \nu /\left(1+n^{2}\right)$, let

$$
\begin{aligned}
& \left|a_{i j}\left(t+s_{p}\right)-a_{i j}\left(t+s_{l}\right)\right| \leq \varepsilon\left(n \sum_{j=1}^{n} H_{j}\right)^{-1}, \\
& \mid \int_{t_{0}}^{t+s_{p}} k_{i j}\left(t+s_{p}, \tau\right) x_{j}(\tau) d \tau \\
& \quad-\int_{t_{0}}^{t+s_{l}} k_{i j}\left(t+s_{l}, \tau\right) x_{j}(\tau) d \tau \mid<\varepsilon .
\end{aligned}
$$

Now, if $t \geq t_{0}$ and $t \in\left[t_{k-1}, t_{k}\right)$ for the upper right-hand derivative ${ }^{c} D_{+}^{q} V\left(x\left(t+s_{p}\right), x\left(t+s_{l}\right)\right)$ along the solutions of system (46), we have

$$
\begin{aligned}
& { }^{c} D_{+}^{q} V\left(x\left(t+s_{p}\right), x\left(t+s_{l}\right)\right) \leq-\sum_{i=1}^{n}\left[c_{i}\right. \\
& \left.-L_{i} \sum_{j=1}^{n}\left|a_{j i}\left(t+s_{p}\right)\right|\right]\left|x_{i}\left(t+s_{p}\right)-x_{i}\left(t+s_{l}\right)\right|
\end{aligned}
$$

$$
\begin{aligned}
& +\sum_{j=1}^{n} \sum_{i=1}^{n}\left|a_{i j}\left(t+s_{p}\right)-a_{i j}\left(t+s_{l}\right)\right| H_{j} \\
& +\sum_{j=1}^{n} \sum_{i=1}^{n} \mid \int_{t_{0}}^{t+s_{p}} k_{i j}\left(t+s_{p}, \tau\right) x_{j}(\tau) d \tau \\
& -\int_{t_{0}}^{t+s_{l}} k_{i j}\left(t+s_{l}, \tau\right) x_{j}(\tau) d \tau \mid \leq-M V\left(x\left(t+s_{p}\right),\right. \\
& \left.x\left(t+s_{l}\right)\right) .
\end{aligned}
$$

Finally, by (50) and (52) it follows that all conditions of Theorem 11 are met. This means that for the impulsive CNN fractional-order integrodifferential system (46) there exists a unique globally perfectly uniform-asymptotically stable almost periodic solution.

\section{Conclusions}

We have considered an impulsive fractional-order integrodifferential system which has a major role in various fields of science and engineering. We first discuss the application of the fractional Lyapunov method in investigations of the existence of almost periodic solutions for such systems. By using a new fractional comparison principle the stability behavior of the almost periodic solution is studied. Some known results are improved and generalized. The demonstrated techniques are applied in studying of qualitative properties of a fractional integrodifferential neural network system.

\section{Conflict of Interests}

The author declares that there is no conflict of interests regarding the publication of this paper.

\section{References}

[1] M. Benchohra, J. Henderson, and S. Ntouyas, Impulsive Differential Equations and Inclusions, Hindawi Publishing Corporation, New York, New York, NY, USA, 2006.

[2] M. de la Sen, "Global stability of polytopic linear time-varying dynamic systems under time-varying point delays and impulsive controls," Mathematical Problems in Engineering, vol. 2010, Article ID 693958, 33 pages, 2010.

[3] I. M. Stamova and T. G. Stamov, "Asymptotic stability of impulsive control neutral-type systems," International Journal of Control, vol. 87, no. 1, pp. 25-31, 2014.

[4] V. Lakshmikantham, M. Rama, and M. Rao, Theory of IntegroDifferential Equations, Gordon and Beach Science Publishers, Lausanne, Switzerland, 1995.

[5] J. J. Nieto and R. Rodriguez-López, "New comparison results for impulsive integro-differential equations and applications," Journal of Mathematical Analysis and Applications, vol. 328, no. 2, pp. 1343-1368, 2007.

[6] G. T. Stamov, Almost Periodic Solutions of Impulsive Differential Equations, Springer, Berlin, Germany, 2012.

[7] I. Stamova, Stability Analysis of Impulsive Functional Differential Equations, Walter De Gruyter, Berlin, Germany, 2009. 
[8] D. Xu, W. Zhu, and S. Long, "Global exponential stability of impulsive integro-differential equation," Nonlinear Analysis, vol. 64, no. 12, pp. 2805-2816, 2006.

[9] B. Ahmad and J. J. Nieto, "Existence of solutions for impulsive anti-periodic boundary value problems of fractional order," Taiwanese Journal of Mathematics, vol. 15, no. 3, pp. 981-993, 2011.

[10] J. Cao and H. Chen, "Impulsive fractional differential equations with nonlinear boundary conditions," Mathematical and Computer Modelling, vol. 55, no. 3-4, pp. 303-311, 2012.

[11] M. Feckan, Y. Zhou, and J. Wang, "On the concept and existence of solution for impulsive fractional differential equations," Communications in Nonlinear Science and Numerical Simulation, vol. 17, no. 7, pp. 3050-3060, 2012.

[12] J. Wang, Y. Zhou, and M. Feckan, "On recent developments in the theory of boundary value problems for impulsive fractional differential equations," Computers and Mathematics with Applications, vol. 64, no. 10, pp. 3008-3020, 2012.

[13] J. R. Wang, M. Fečkan, and Y. Zhou, "Ulam's type stability of impulsive ordinary differential equations," Journal of Mathematical Analysis and Applications, vol. 395, no. 1, pp. 258-264, 2012.

[14] G. T. Stamov and I. M. Stamova, "Almost periodic solutions for impulsive fractional differential equations," Dynamical Systems, vol. 29, no. 1, pp. 119-132, 2014.

[15] F. Chen, A. Chen, and X. Wang, "On the solutions for impulsive fractional functional differential equations," Differential Equations and Dynamical Systems, vol. 17, no. 4, pp. 379-391, 2009.

[16] T. L. Guo and W. Jiang, "Impulsive fractional functional differential equations," Computers \& Mathematics with Applications, vol. 64, no. 10, pp. 3414-3424, 2012.

[17] H. Wang, "Existence results for fractional functional differential equations with impulses," Journal of Applied Mathematics and Computing, vol. 38, no. 1-2, pp. 85-101, 2012.

[18] I. Stamova and G. Stamov, "Stability analysis of impulsive functional systems of fractional order," Communications in Nonlinear Science and Numerical Simulation, vol. 19, no. 3, pp. 702-709, 2014.

[19] A. Anguraj and M. L. Maheswari, "Existence of solutions for fractional impulsive neutral functional infinite delay integrodifferential equations with nonlocal conditions," Journal of Nonlinear Science and its Applications, vol. 5, pp. 271-280, 2012.

[20] Z. Gao, L. Yang, and G. Liu, "Existence and uniqueness of solutions to impulsive fractional integro-differential equations with nonlocal conditions," Applied Mathematics, vol. 4, no. 6, pp. 859-863, 2013.

[21] S. Xie, "Existence results of mild solutions for impulsive fractional integro-differential evolution equations with infinite delay," Fractional Calculus and Applied Analysis, vol. 17, no. 4, pp. 1158-1174, 2014.

[22] I. Podlubny, Fractional Differential Equations, Mathematics in Science and Engineering, Academic Press, San Diego, Calif, USA, 1999.

[23] V. Lakshmikantham, S. Leela, and M. Sambandham, "Lyapunov theory for fractional differential equations," Communications in Applied Analysis, vol. 12, no. 4, pp. 365-376, 2008. 


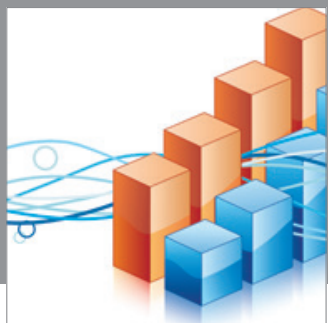

Advances in

Operations Research

mansans

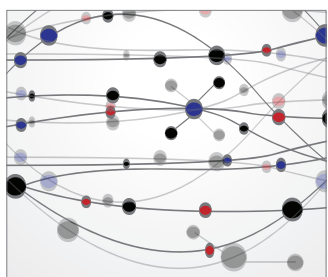

The Scientific World Journal
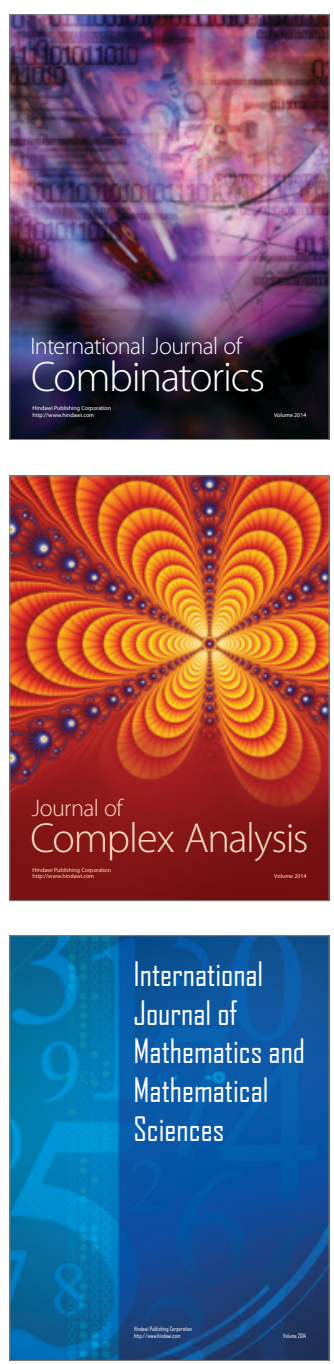
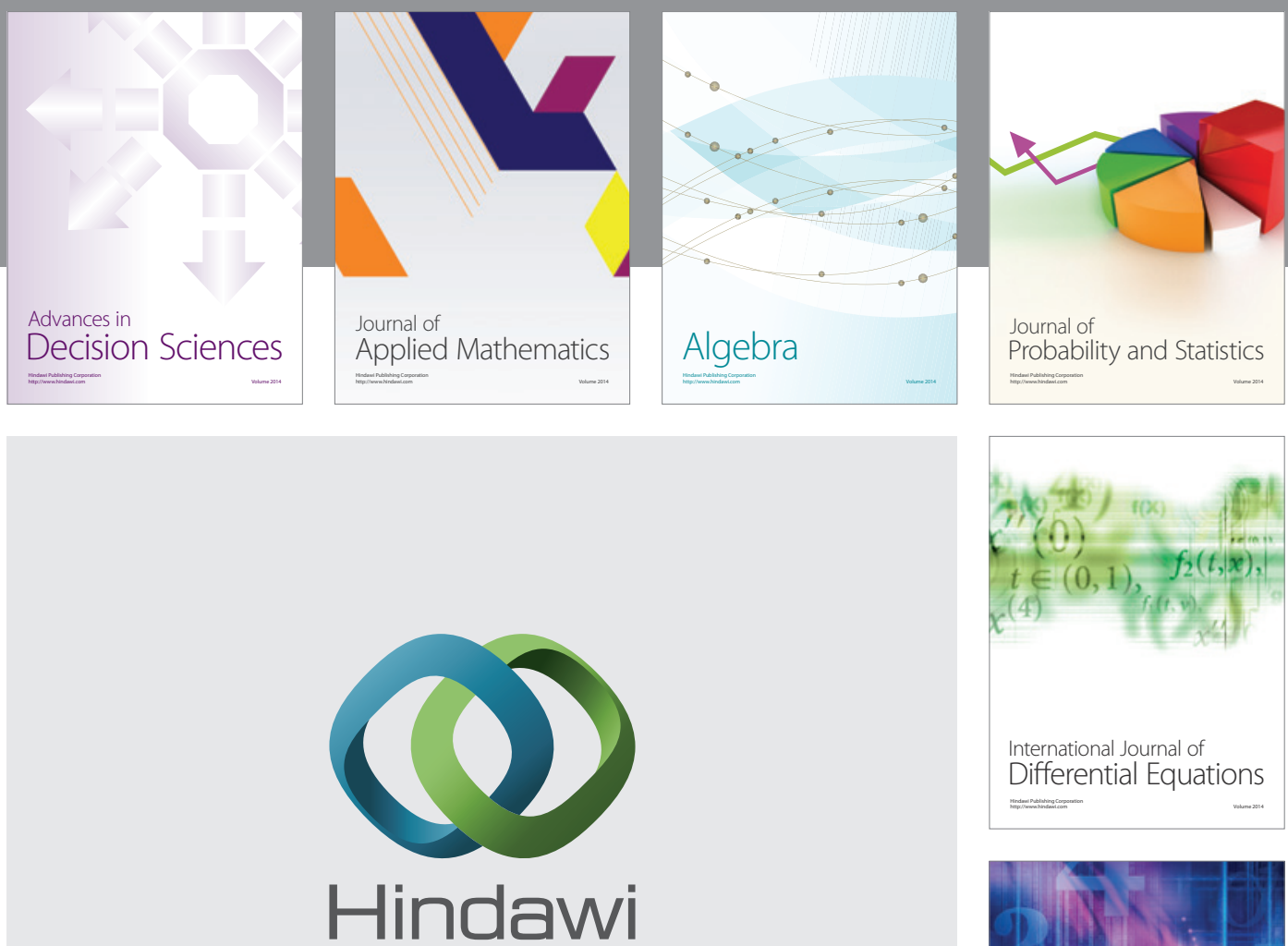

Submit your manuscripts at http://www.hindawi.com
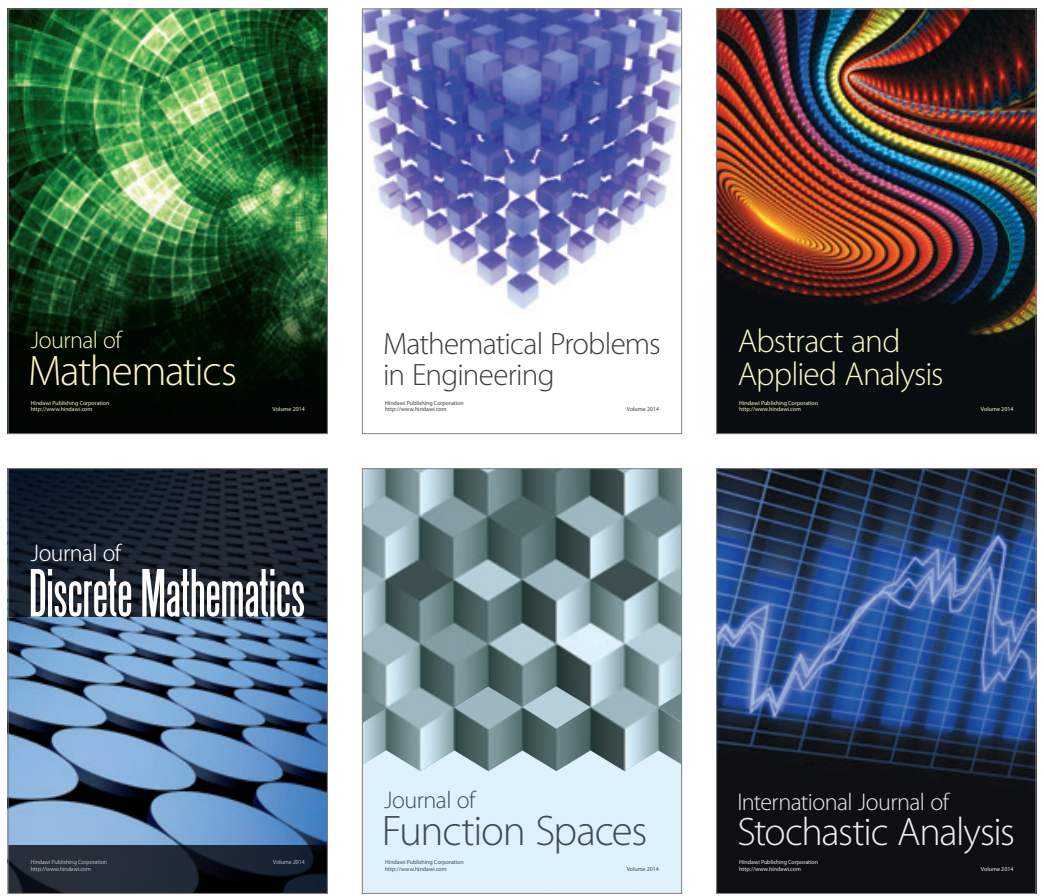

Journal of

Function Spaces

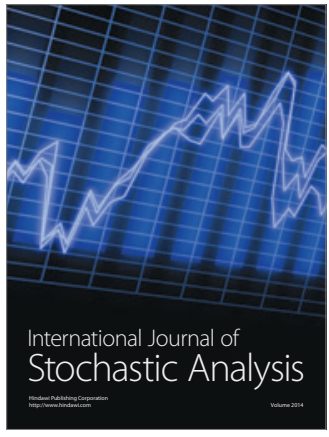

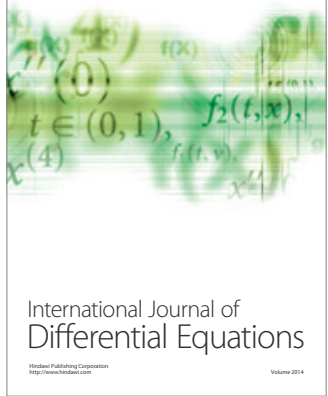
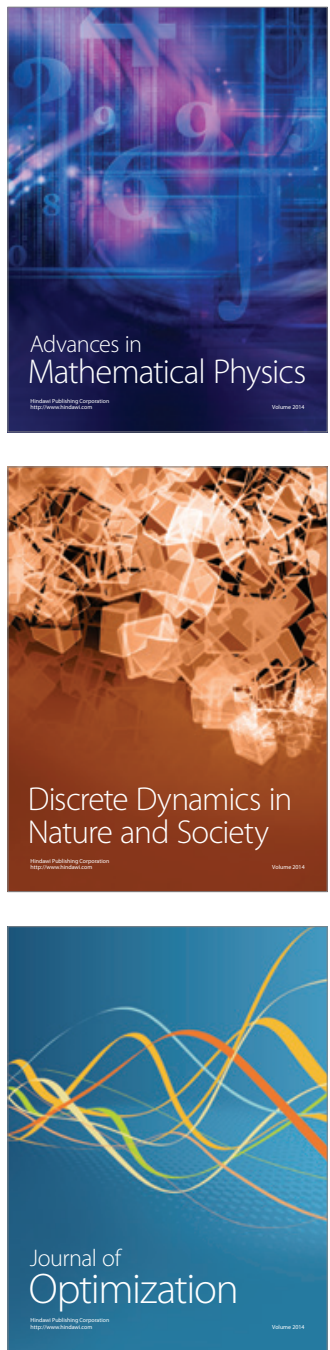